Submission ID: 43845

\title{
Seismic Non-Linearity in Case of Single Shot Impact. Matched Non-Linear
} Operator Calculation

A.A. Arkhipov* (Geoshelf-Service), V.S. Tyrichev (Geoshelf-Service)

\section{SUMMARY}

Significant non-linear effects of geological environment in seismic data are obvious in case of vibration impact. Authors described their version of non-linearity explanation in case of single shot impact. First time a method of linearization of seismic data introduced. Method allows noticeably rise correlativity between linear-modelled data and conventional seismic. 
Сейсмическая нелинейность при одиночном импульсном возбуждении. Практика применения согласованного нелинейного оператора.

\author{
А.А.Архипов* (ООО «Геошельф-Сервис»), В.С.Тыричев (ООО «Геошельф-Сервис»)
}

\title{
Введение
}

Существования значимой нелинейности геологической среды в сейсмическом диапазоне частот доказано рядом авторов полевыми работами при возбуждении вибраторами воздействий близких к монохроматическим. Для исключения влияния нелинейности системы возбуждения и нелинейности, возникающей на контакте «плита-грунт» вибраторы работали в паре при существенных удалениях друг от друга. Перед непосредственной работой проводилась регистрация нелинейных (механических) сигнатур самих установок.

Ранее авторами доклада было получено подтверждение гипотезы о значимой нелинейности геологической среды при широкополосном воздействии на среду «точечным» источником. На примере данных морской сейсморазведки произведено восстановление предварительно исключённой НЧ записи волнового поля применением обратного нелинейного оператора, инвертирующего «след» перераспределения энергии НЧ в область ВЧ.

В настоящей работе рассматриваются принцип формирования нелинейного отклика среды при одноточечном воздействии с позиции дисперсии скоростей распространения упругих волн и частично упругом взаимодействии.

В заключении приводится пример применения согласованного нелинейного оператора в сравнении с линейным согласованным фильтром для цели скважинно-ориентированной обработки данных сейсморазведки.

\section{Теория}

В случае частично упругого взаимодействия возникают деформационные колебания, затухающие со временем (переход энергии в тепло), после чего тело принимает прежнюю форму. При этом мода частоты осцилляций тела, которому передаётся энергия, соответствует частоте воздействия.

В нелинейной акустике считается, что нелинейность обусловлена разницей в скоростях перемещения точек профиля фронта волны. Иными словами, скорость пробега упругих волн увеличивается в областях сжатия и уменьшается в областях разряжения.

Ряд исследований, с использованием двух удалённых друг от друга вибрационных источников, не противоречит предыдущему утверждению (Николаев А.В. и др., 1987, Логинов К.И., Жуков А.П., Шнеерсон М.Б, Логинов И.В., 2012), т.к. время подхода фронта воздействия на отражающую границу от каждого источника - различно.

Возникает закономерный вопрос: каким образом объяснить значимую нелинейность (Архипов А.А., Тыричев В.С., 2017), регистрируемую при одиночном источнике/синхронизированной группе?

Достаточно лаконичный ответ на этот вопрос можно получить при рассмотрении дисперсии скоростей пробега упругих волн сейсмического диапазона частот. На рисунке 1 изображены зависимости групповых и фазовых скоростей пробега продольной и поперечной волн от частоты (для пористого образца). 
Строго говоря, в случае вибрационного монохроматического возбуждения (генерации управляющего монохроматического сигнала), среда зондируется почти монохроматическими волнами, для которых справедлива групповая (но достаточно узкополосная) оценка скорости пробега упругих волн, приближенная к фазовой.

При импульсном возбуждении, в следствии дисперсии скоростей пробега упругих волн, со временем цуг зондирующего широкополосного воздействия «удлиняется» во времени (низкочастотные компоненты запаздывают, относительно высокочастотных). Следовательно, в первую очередь на пласт происходит высокочастотное воздействие, а уже после низкочастотное.
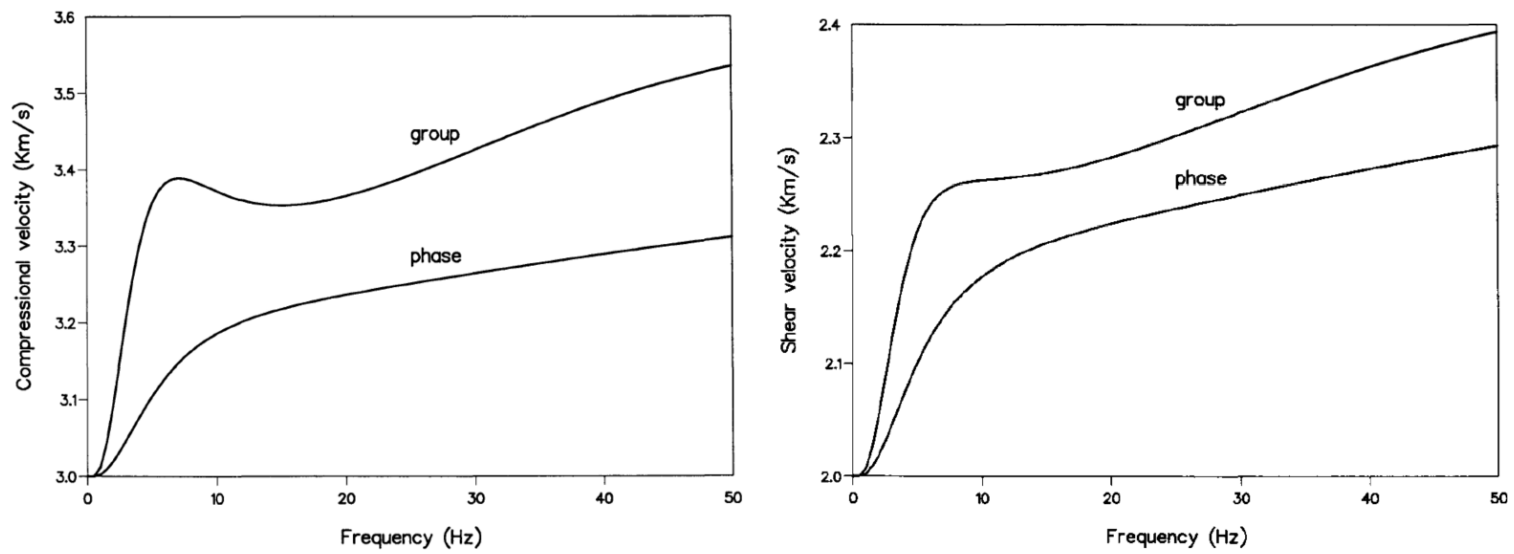

Рисунок 1 Дисперсия скоростей пробега упругих волн в сейсмическом диапазоне частот для пористого образияа горной породы (Jose M. Carcione, 1988).

Таким образом, принципы, положенные в основу анализа нелинейных эффектов при монохроматических (вибрационных) воздействиях на среду справедливы для случая импульсного источника.

Из практики вибрационной сейсморазведки известно, что при воздействии на пласт двумя (и более) монохроматическими сигналами, в силу нелинейности, в спектре отклика регистрируются комбинационные частоты (аддитивные, кратные, комбинационные и т.д.). Следовательно при широкополосном воздействии на поверхности регистрируется спектр нелинейного отклика (в частности отражённых волн) с перераспределённой энергией.

Логично предположить, что при дальнейшем комплексировании материалов сейсморазведки со скважинными данными на этапе инверсионных преобразований или скважинно-ориентированной обработки полевых данных, необходимо работать с линейным откликом среды (либо учитывать нелинейность на протяжении обработки и интерпретации, включая стадию моделирования).

Очевидно, что нелинейность является одной из причин несогласованности результатов одномерного моделирования (линейного) и сейсмических суммотрасс. Для увеличения коррелятивности и, как следствие, уменьшения ошибки инверсии авторы предлагают применять согласованный нелинейный оператор (линеаризующий). При постобработке оператор рассчитывается после привязки скважинных данных к результирующему временному разрезу, в случае обработки полевых данных к предварительной сумме.

\section{Примеры}

Применение нелинейного оператора неизбежно приводит к перераспределению энергии зарегистрированного спектра, в частности, к его расширению в области низких и высоких 


\section{EAGE}

частот. Однако, в текущей работе будет рассмотрено сопоставление результатов применения согласованного (линейного) фильтра и согласованного нелинейного оператора к мигрированным суммам при одинаковых условиях поиска решения (в том числе по частотам).

Для примера рассмотрим сравнение результатов применения согласованных преобразований в случае донной сейсморазведки по обменной (PSv) компоненте (Рисунки 2-5).

Согласованные операции рассчитывались в 2.5сек интервале с контролем общего и частного (в целевом диапазоне) коэффициентов взаимной корреляции результирующей и синтетической суммотрасс с дополнительной проверкой результатов инверсии (Таблица 1).

Все разрезы, включая разностный, изображены в единой шкале амплитуд.

\begin{tabular}{|l|l|l|l|l|l|l|}
\hline & Исх.Ккорр & Рез.Ккорр & Рез.инверсии & Исх.Ккорр & Рез.Ккорр & Рез.инверсии \\
\hline Линейный & 0.652 & 0.721 & 0.705 & 0.629 & 0.687 & 0.743 \\
\hline Нелинейный & 0.652 & 0.865 & 0.853 & 0.629 & 0.860 & 0.881 \\
\hline
\end{tabular}

Таблица 1 Количественная оценка результата применения согласованных операций.

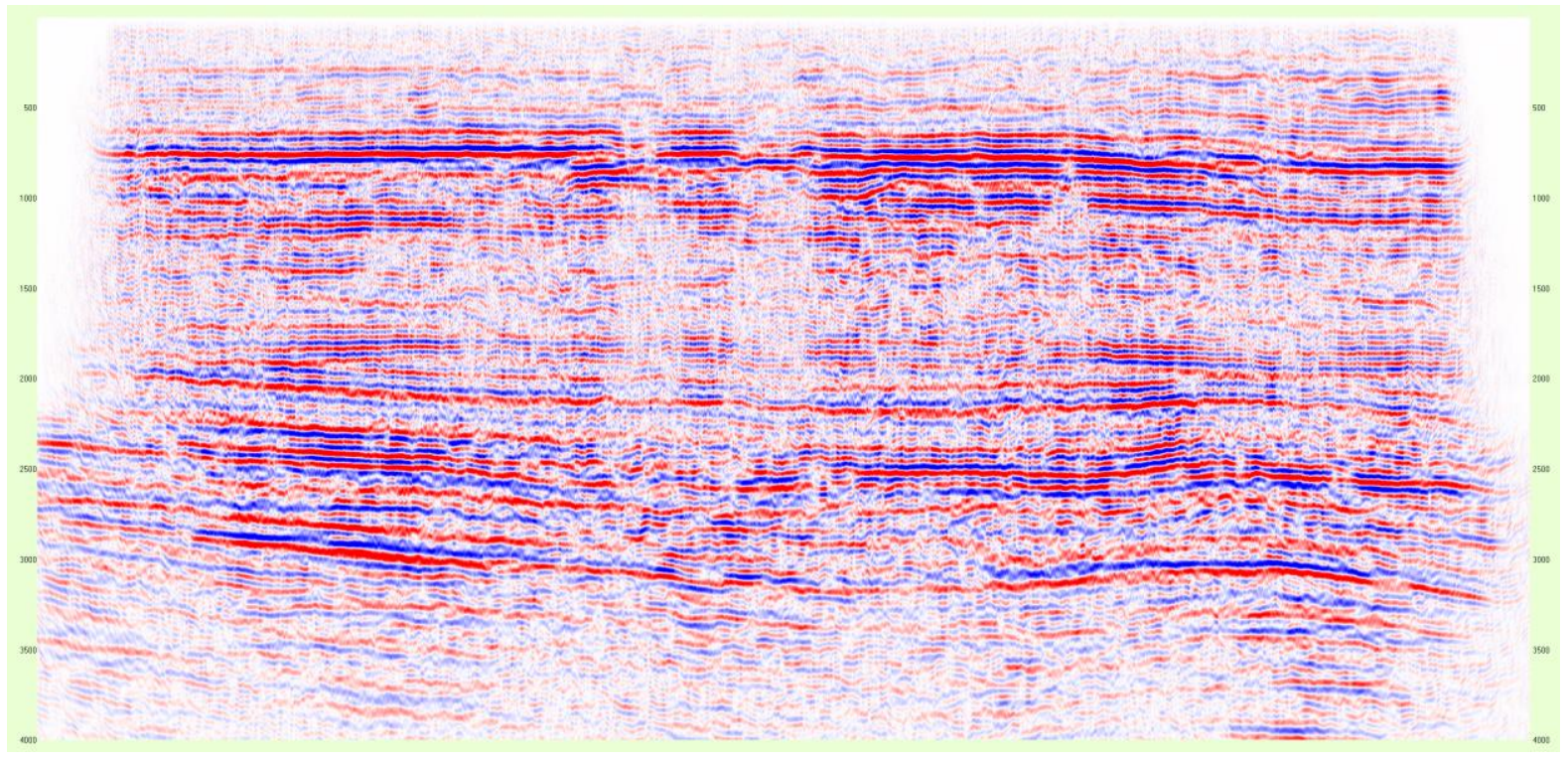

Рисунок 2 Разрез PSv до применения согласованных операщий.

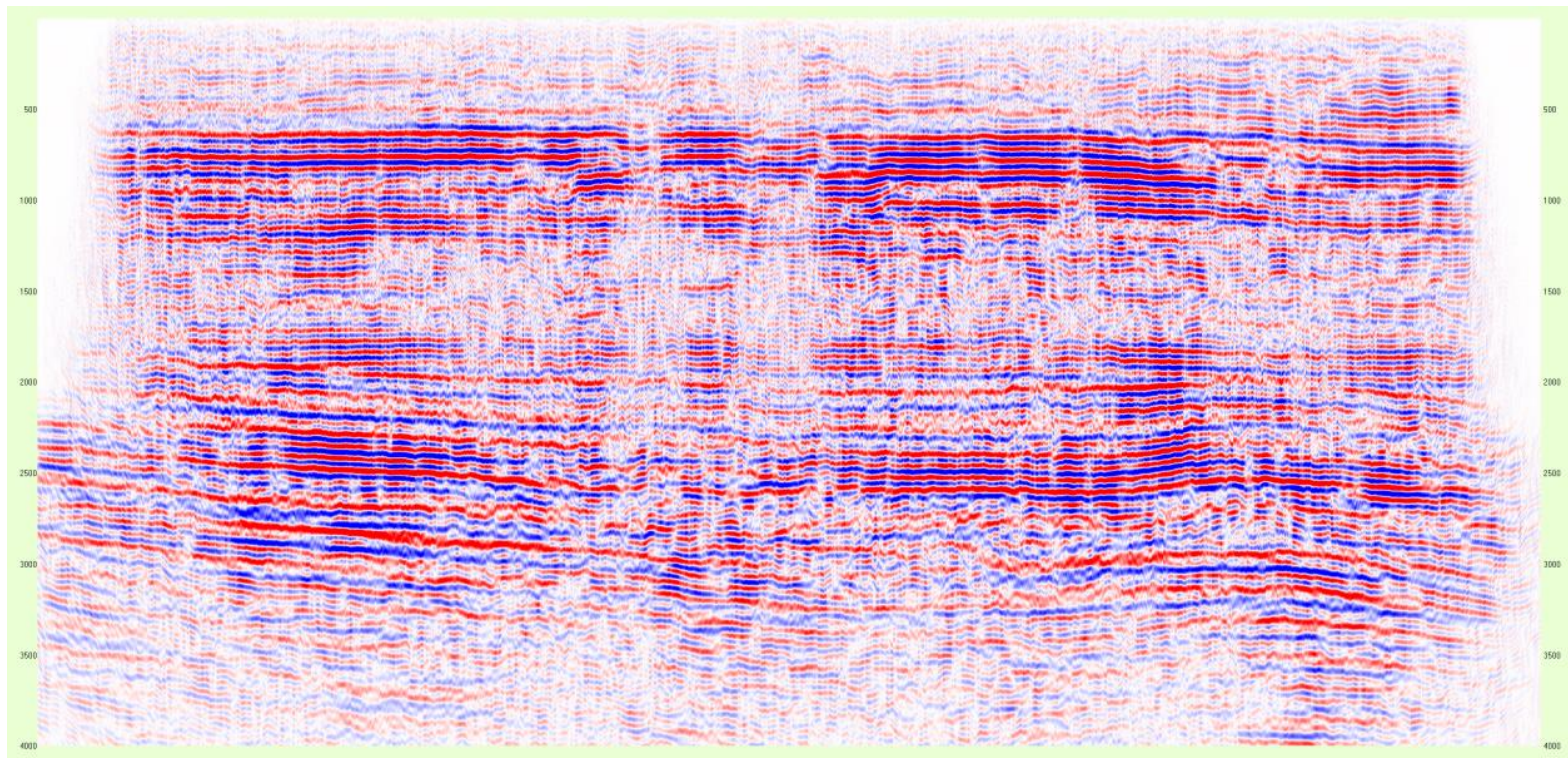

Рисунок 3 Разрез PSv после применения согласованного (линейного) фильтра. 


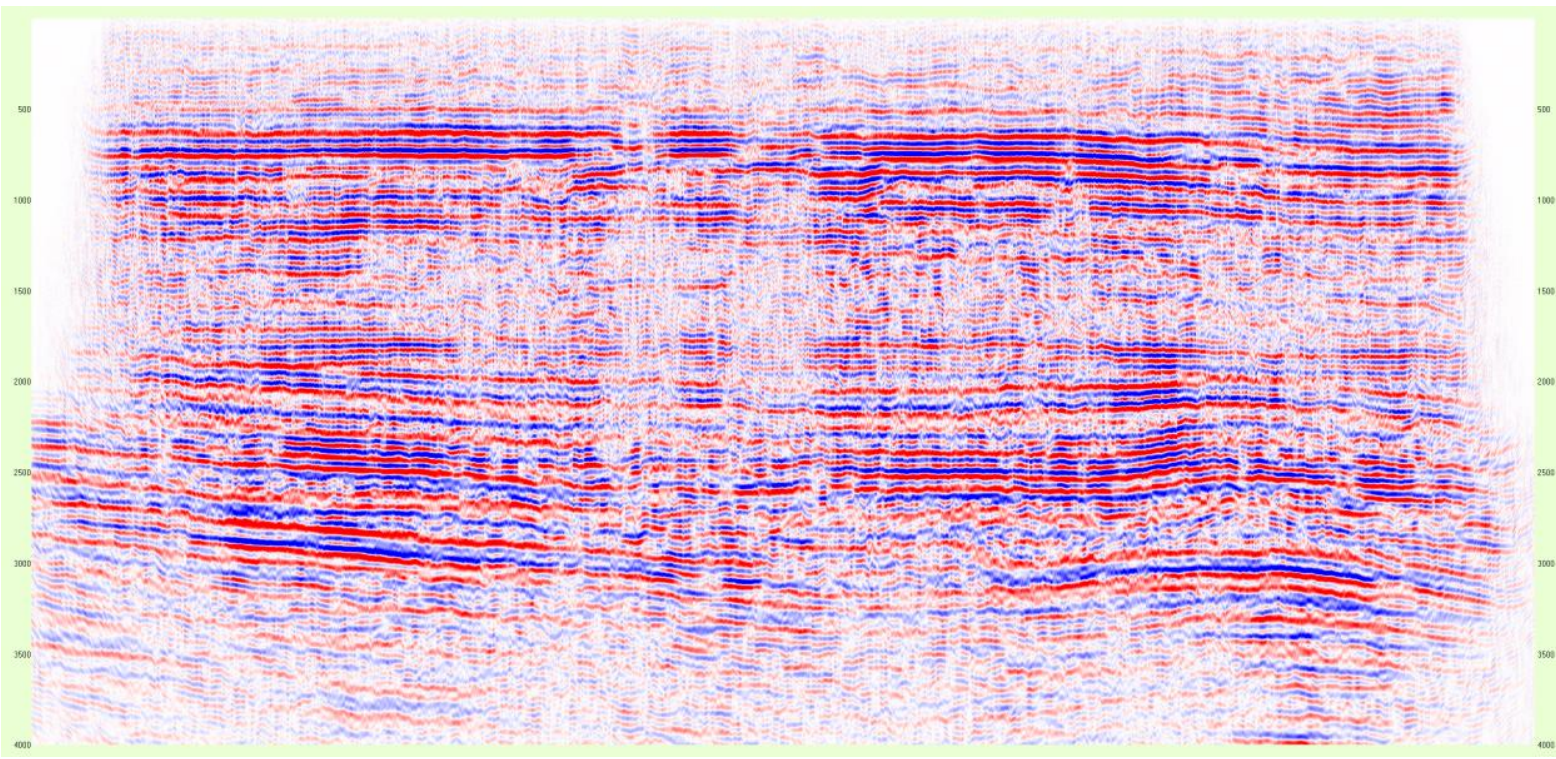

Рисунок 4 Разрез PSv после применения согласованного нелинейного оператора.

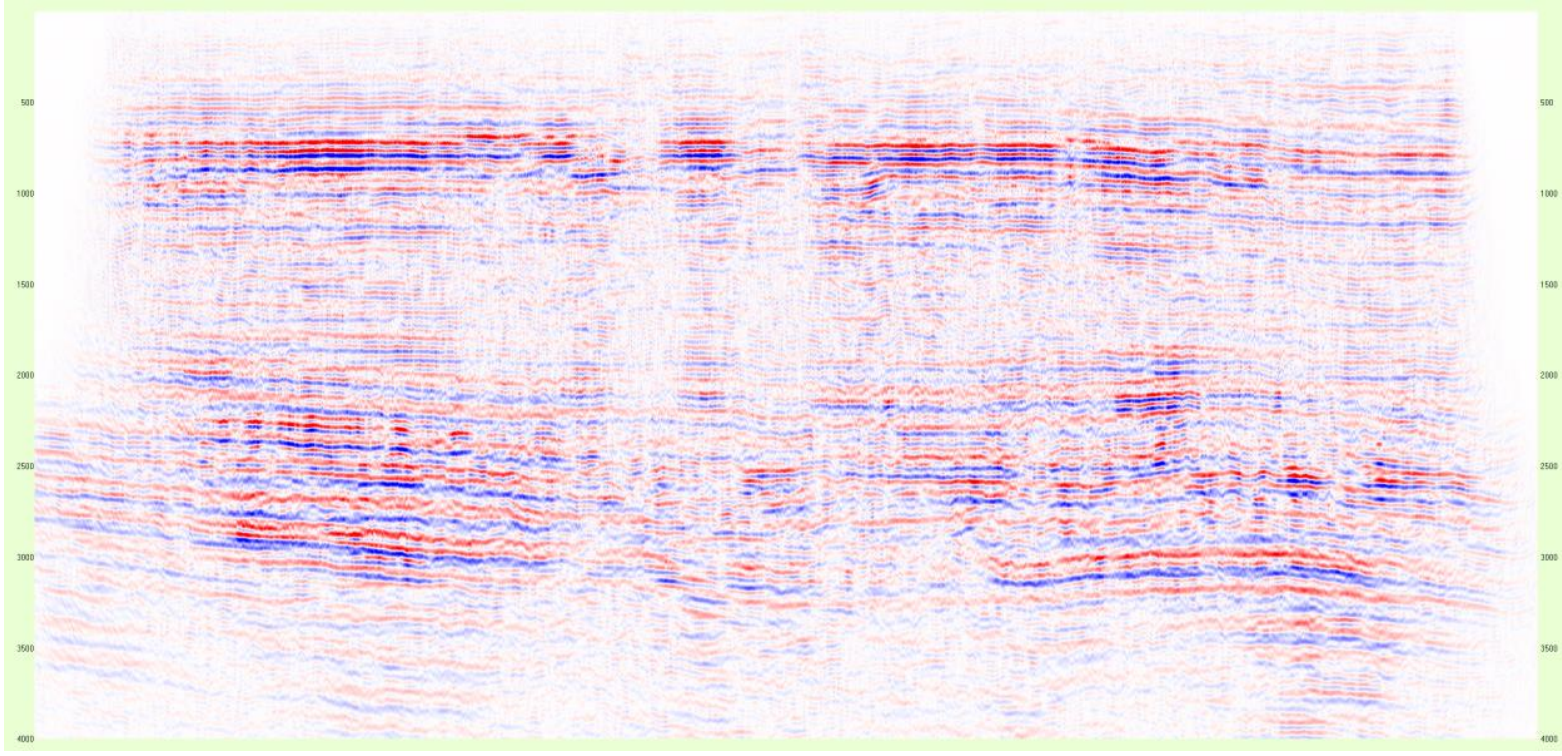

Рисунок 5 Разрез PSv. Разность применения линейного и нелинейного согласования.

\section{Выводы}

Авторами предложен вариант модели генерации нелинейных компонент волнового поля при одиночном импульсном воздействии на среду.

Разработан и применён модуль расчёта согласованного нелинейного оператора (линеаризующего), позволяющий существенно повысить коррелятивность с данными линейного моделирования и инверсионных преобразований.

\section{Библиография}

Николаев А.В. [1987] Проблемы нелинейной сейсмики. Наука Логинов К.И., Жуков А.П., Шнеерсон М.Б, Логинов И.В. [2012] Нелинейные волновые поля в акустическом каротаже и вибрационной сейсморазведке. ГЕРС

Архипов А.А., Тыричев В.С. [2017] О существовании значимой нелинейности в сейсмическом диапазоне частот. Часть 1 . Тезисы «Геосочи-2017», с. 150-157 


\section{EAGE}

\section{References}

Nikolaev A.V. [1987] Problems of non-linear seismic. Nauka

Loginov K.I., Zhukov A.P., Shneerson M.B, Loginov I.V. [2012] Non-linear wavefields in sonic well data and vibration seismic. Gers

Arkhipov A.A., Tyrichev V.S. [2017] About existence of significant non-linearity in seismic frequency band. Part 1. Thesis «Geosochi-2017», c. 150-157 